\title{
The Cohomology of Nets over Minkowski Space
}

\author{
P. Leyland ${ }^{1}$ and J. E. Roberts ${ }^{2}$ \\ 1 Centre de Physique Théorique, CNRS, F-13274 Marseille Cedex 2, France \\ 2 Fachbereich 5, Universität Osnabrück, D-4500 Osnabruck, Federal Republic of Germany
}

\begin{abstract}
We investigate the cohomology of nets over Minkowski space and develop exact sequence techniques enabling us to compute many lowdimensional cohomologies. We examine in particular nets derived from smooth solutions of invariant partial differential equations using causal support conditions. Thus the wave equation gives a trivial second cohomology whereas the vector wave equation with Lorentz condition and Maxwell's equations give a second cohomology $\mathbb{R}$ and $\mathbb{R} \times \mathbb{R}$ corresponding, respectively, to an electric and an electric and magnetic charge.
\end{abstract}

\section{Introduction}

This paper has its origins in investigations into the structure of quantum field theory. In classical (relativistic) field theory, a field can be thought of as a function $\phi(x)$ defined on space-time (Minkowski space) with values in the real line or, more ambitiously, in other manifolds. The set of fields at time $x^{0}=0$ constitute the infinite-dimensional configuration space of the system. If the fields at time $x^{0}=0$ are taken together with their conjugate momenta in the sense of Lagrangian field theory, we get the infinite-dimensional phase space of the system. Quantum fields are too singular to admit any such interpretation; they are distributions rather than functions. In any case, quantum theory does not deal directly with configuration spaces or phase spaces but instead real-valued functions on configuration space are replaced by commuting self-adjoint operators on a Hilbert space and real-valued functions on phase space by non-commuting self-adjoint operators.

A rigorous mathematical framework for quantum field theory was given by Gårding and Wightman [1,2] who considered quantum fields to be operatorvalued distributions. Here the basic objects are unbounded self-adjoint operators

$$
\phi(f)=\int \phi(x) f(x) d x,
$$

where $f$ is a smooth function of compact support on Minkowski space. To relate this with the above ideas, $f$ should be regarded as a linear function on the (linear) phase space of the system. 
Algebraic field theory, initiated by Haag [3], makes a radical break with the ideas of conventional field theory. The basic mathematical object is a net of von Neumann algebras $\mathcal{O} \rightarrow \mathfrak{U}(\mathcal{O})$ on Minkowski space. Here $\mathcal{O}$ is a subset of Minkowski space and the relation with Wightman field theory is that $\mathfrak{A}(\mathcal{O})$ is the von Neumann algebra generated by the bounded functions of the self-adjoint operators $\phi(f)$, supp $f \subset \mathcal{O}$. Thus the fields are relegated to the role of being one possible system of "generators" for the algebras $\mathfrak{A}(\mathcal{O})$. All that remains of the dependence of fields on a space-time point is the net structure, i.e. the dependence of the algebras $\mathfrak{A}(\mathcal{O})$ on the set $\mathcal{O}$.

Considering the radical nature of these proposals, algebraic field theory has been remarkably successful in elucidating the structural features of quantum field theory. It has recently become clear $[4,5]$ that some of the most interesting structural features are related to a novel form of cohomology of the net $\mathcal{O} \rightarrow \mathfrak{A}(\mathcal{O})$ called local cohomology. Unfortunately, as the algebras $\mathfrak{U}(\mathcal{O})$ are not Abelian, this cohomology is a non-Abelian cohomology, so that many of the useful techniques of the Abelian cohomology are not immediately available. For this reason, it seemed sensible to study an analogue of local cohomology in an Abelian setting. This is what is done in this paper by replacing the net $\mathcal{O} \rightarrow \mathfrak{U}(\mathcal{O})$ by a net of Abelian groups.

In Section 1 we formulate local cohomology as a cohomology for nets of Abelian groups defined on the set of compact sets in Minkowski space. Now nets of Abelian groups do arise in quantum field theory if one considers the net $\mathcal{O} \rightarrow \mathfrak{B}(\mathcal{O})$ where $\mathfrak{B}(\mathcal{O})$ is the group of inner automorphisms of the free field algebra generated by the Weyl operators $e^{i \phi(f)}, \operatorname{supp} f \subset \mathcal{O}$. The groups $\mathfrak{B}(\mathcal{O})$ can be identified with the set of solutions of the corresponding free field equation (e.g. the wave equation) whose Cauchy data have support in $\mathcal{O}$. This motivates the study in Section 2 of nets $\mathfrak{B}$ constructed from sheaves of Abelian groups by letting $\mathfrak{B}(\mathcal{O})$ denote the group of global sections that coincide with the zero section on the spacelike complement of $\mathcal{O}$. The most important result here is Theorem 2.10 which states that the second cohomology is trivial if the sheaf is causally soft. A sheaf is causally soft either if it is soft itself or if there is an associated soft sheaf of Cauchy data. Theorem 2.10 shows in particular that the net of smooth solutions of the wave equation or of the Klein-Gordon equation has a trivial second cohomology.

As might be expected, the basic technique for computing non-trivial cohomologies is to exploit exact sequences. Unfortunately, the sequences of nets arising in practice are rarely exact as they stand but they often become exact if the nets are restricted to a subset of the compact sets, the set of double cones. We show in Theorem 3.1, by using barycentric decompositions of simplexes that the cohomology depends only on its restriction to the set of double cones. In Theorem 3.2 we can then show that a short exact sequence of nets over double cones gives rise to a long exact sequence in cohomology. This enables us to compute further cohomologies. In particular we prove that the vector wave equation with Lorentz condition has $\mathbb{R}$ as its second cohomology where the parameter may be interpreted as an electric charge. Maxwell's equations have $\mathbb{R} \times \mathbb{R}$ as the second cohomology where the parameters can be interpreted as an electric and a magnetic charge. 


\section{Net Cohomology}

We define a cohomology for nets of Abelian groups over the set $\mathscr{C}$ of compact subsets in Minkowski space ordered under inclusion. $\mathfrak{A}$ is said to be a net of Abelian groups if $\mathfrak{A}(F)$ is an Abelian group for each $F \in \mathscr{C}$ and if $F_{1} \subset F_{2}$ implies that $\mathfrak{A}\left(F_{1}\right)$ is a subgroup of $\mathfrak{Q}\left(F_{2}\right)$. Thus a net is simply a strict inductive system in which $\mathscr{U}(F)$ has been identified with the corresponding subgroup of the inductive limit. Unless otherwise stated a net will mean a net of Abelian groups. Any Abelian group $A$ can and will be considered as a trivial net, also denoted by $A$, by setting $A(F)=A, F \in \mathscr{C}$.

The nets of most interest to us here are those which reflect the causal structure of Minkowski space $\mathbb{R}^{s+1}$. This causal structure is defined in terms of the quadratic form $(x, x)=\left(x^{0}\right)^{2}-\sum_{i=1}^{s}\left(x^{i}\right)^{2} . x$ and $y$ are said to be timelike, lightlike or spacelike according as $(x-y)^{2}>0,(x-y)^{2}=0,(x-y)^{2}<0$. Let $V_{+}=\left\{x: x^{0} \geqq 0\right.$ and $(x, x) \geqq 0\}$. If $x-y \in V_{+}$and $x \neq y$, then $\mathcal{C}=\left(x-V_{+}\right) \cap\left(y+V_{+}\right)$is said to be the double cone with vertices $x$ and $y$. It is said to be based on the spacelike hyperplane $H=\left\{x^{\prime} \in \mathbb{R}^{s+1}:\left(x^{\prime}-\frac{1}{2}(x+y), x-y\right)=0\right\}$ and to have base $H \cap \mathcal{O}$. Let $\mathscr{K}$ denote the set of double cones ordered under inclusion and $\mathscr{K}_{0}$ the subset of double cones centred on the origin, i.e. with $y=-x$.

Let $\Sigma_{n}$ denote the set of singular $n$-simplexes in Minkowski space, i.e. the set of continuous maps from the standard $n$-simplex

$$
\Delta^{n}=\left\{\left(t^{0}, t^{1}, t^{2}, \ldots t^{n}\right) \in \mathbb{R}^{n+1}: t^{i} \geqq 0, \sum_{i=0}^{n} t^{i}=1\right\}
$$

into Minkowski space. There are face maps $\partial_{i}: \Sigma_{n} \rightarrow \Sigma_{n-1} i=0,1, \ldots n$ defined by

$$
\left(\partial_{i} c\right)\left(t^{0}, t^{1}, \ldots t^{n-1}\right)=c\left(t^{0}, t^{1}, \ldots t^{i-1}, 0, t^{i}, \ldots, t^{n-1}\right), c \in \Sigma_{n}
$$

and degeneracy maps $\sigma_{i}: \Sigma_{n} \rightarrow \Sigma_{n+1}, i=0,1,2, \ldots n$ defined by

$$
\left(\sigma_{i} c\right)\left(t^{0}, t^{1}, \ldots t^{n+1}\right)=c\left(t^{0}, t^{1}, \ldots, t^{i-1}, t^{i}+t^{i+1}, t^{i+2}, \ldots, t^{n+1}\right), c \in \Sigma_{n} .
$$

An $n$-cochain with values in a net $\mathfrak{A}$ is a map $f: \Sigma_{n} \rightarrow \bigcup_{F \in \mathscr{C}} \mathfrak{A}(F)$ such that there exists an $\mathscr{O} \in \mathscr{K}_{0}$ with

$$
f(c) \in \mathfrak{A}(\mathcal{O}+|c|) \quad c \in \Sigma_{n} .
$$

Here $|c|=c\left(\Delta^{n}\right)$ is the support of $c$. (1.1) is the critical "locality" condition on the $n$ cochain which says that $f(c)$ is localized about $|c|$ to within some uniform error $\mathcal{U}$. The choice of $\mathscr{K}_{0}$ is dictated by convenience and could be replaced by any other cofinal subset of $\mathscr{C}$. Since $\mathcal{O}+\left|\partial_{i} c\right| \subset \mathcal{O}+|c|$, we may define an $(n+1)$-cochain $d f$ by

$$
d f(c)=\sum_{i=0}^{n+1}(-1)^{i} f\left(\partial_{i} c\right)
$$

and get in this way a cochain complex $C^{*}(\mathfrak{H})$ of Abelian groups,

$$
C^{0}(\mathfrak{U}) \stackrel{d}{\longrightarrow} C^{1}(\mathfrak{U}) \stackrel{d}{\longrightarrow} C^{2}(\mathfrak{U}) \rightarrow \ldots
$$


The cohomology of the net $\mathfrak{A}$ is the cohomology of this cochain complex and we write as usual $Z^{n}(\mathfrak{U})=\operatorname{Ker} d, B^{n}(\mathfrak{U})=\operatorname{Im} d$, with the convention that $B^{0}(\mathfrak{U})=0$, and $H^{n}(\mathfrak{U})=Z^{n}(\mathfrak{A}) / B^{n}(\mathfrak{U})$. If we want to indicate a specific choice of $\mathscr{O} \in \mathscr{K}_{0}$ in (1.1), we write $C^{n}(\mathcal{O} ; \mathfrak{A}), Z^{n}(\mathcal{O} ; \mathfrak{U})$ etc. The cohomology of a net $\mathfrak{A}$ is unchanged if we replace it by its outer regularization $\hat{\mathfrak{U}}$ or inner regularization $\mathfrak{\mathfrak { U }}$ defined by

$$
\begin{aligned}
& \hat{\mathfrak{U}}(F)=\bigcap\left\{\mathfrak{U}\left(F_{1}\right): F\left(\operatorname{int} F_{1}\right\},\right. \\
& \mathfrak{\mathfrak { U }}(F)=\bigcup\left\{\mathfrak{U}\left(F_{1}\right): F_{1} \operatorname{int} F\right\} .
\end{aligned}
$$

If $\mathfrak{U}$ and $\mathfrak{B}$ are nets then a morphism $\phi: \mathfrak{U} \rightarrow \mathfrak{B}$ of nets is of course a set of homomorphisms $\phi(F): \mathfrak{U}(F) \rightarrow \mathfrak{B}(F), F \in \mathscr{C}$, so that if $F_{1} \subset F_{2}$ then $\phi\left(F_{1}\right)$ is the restriction of $\phi\left(F_{2}\right)$ to $\mathfrak{A}\left(F_{1}\right)$. The above definitions are functorial so that $\phi$ induces a morphism $\phi_{*}: C^{*}(\mathfrak{U}) \rightarrow C^{*}(\mathfrak{B})$, where $\phi_{*}(f)(c)=\phi(f(c))$, of the corresponding cochain complexes and thus homophormisms of the cohomology groups. A sequence of nets

$$
0 \rightarrow \mathfrak{A} \stackrel{\phi}{\longrightarrow} \mathfrak{B} \stackrel{\psi}{\longrightarrow} \mathfrak{C} \rightarrow 0
$$

is said to be a short exact sequence if

$$
0 \rightarrow \mathfrak{U}(F) \stackrel{\phi(F)}{\longrightarrow} \mathfrak{B}(F) \stackrel{\psi(F)}{\longrightarrow} \mathfrak{C}(F) \rightarrow 0
$$

is a short exact sequence of Abelian groups for each $F \in \mathscr{C}$.

1.1. Lemma. The short exact sequence (1.5) of nets gives rise to a short exact sequence of cochain complexes and hence to a long exact sequence

$$
0 \rightarrow H^{0}(\mathfrak{U}) \rightarrow H^{0}(\mathfrak{B}) \rightarrow H^{0}(\mathfrak{C}) \rightarrow H^{1}(\mathfrak{U}) \rightarrow H^{1}(\mathfrak{B}) \rightarrow H^{1}(\mathfrak{C}) \rightarrow H^{2}(\mathfrak{U}) \rightarrow \ldots
$$

of cohomology groups.

Proof. A trivial direct computation shows that

$$
0 \rightarrow C^{n}(\mathfrak{U}) \stackrel{\phi_{*}}{\longrightarrow} C^{n}(\mathfrak{B}) \stackrel{\psi_{*}}{\longrightarrow} C^{n}(\mathfrak{C}) \rightarrow 0
$$

is exact for each $n$ and the long exact sequence (1.6) is a standard consequence [6, $\S 2.2]$.

There are two simple general results on net cohomology that follow from the contractibility of Minkowski space. First for any net $\mathfrak{A}$

$$
H^{0}(\mathfrak{U})=\bigcup_{\mathscr{O} \in \mathscr{K}_{0}} \bigcap_{a \in \Sigma_{0}} \mathfrak{U}(\mathcal{O}+a) .
$$

Secondly, if $A$ is a trivial net, $A(F)=F, F \in \mathscr{C}$, the cohomology of $A$ coincides with the cohomology of Minkowski space with values in the Abelian group $A$. Thus $H^{0}(A)=A$ and $H^{n}(A)=0, n \geqq 1$.

Our aim in the following sections is to learn how to compute the cohomology of nets which reflect the causal structure on Minkowski space with a view to developing techniques that can be used in the non-Abelian setting of quantum field theory. We have not attempted to develop a general theory of net cohomology although some further general results can be found in Section 3. The 
category of Abelian groups can of course be replaced by any other Abelian category. Indeed our examples of nets will be almost exclusively nets of $\mathbb{R}$ modules. To what extent net cohomology could be usefully extended to spaces other than Minkowski space is not clear but topology and a concept of uniform boundedness are involved in its definition.

\section{Nets Derived from Sheaves}

One obvious way of constructing nets is to use support properties so that $f \in \mathfrak{U}(F)$ means that $f$ has its support in $F$. To this end, we consider a sheaf $\mathscr{A}$ of Abelian groups on Minkowski space so that $\mathscr{A}(U)$ is an Abelian group for each open set $U$. If $f \in \mathscr{A}\left(\mathbb{R}^{s+1}\right)$, we write $f=0$ on $U$ to mean that $f$ is in the kernel of the restriction map from $\mathscr{A}\left(\mathbb{R}^{s+1}\right)$ to $\mathscr{A}(U)$. We define a net $\Gamma_{b}(\mathscr{A})$ over $\mathscr{C}$ by setting

$$
\Gamma_{b}(\mathscr{A})(F)=\left\{f \in \mathscr{A}\left(\mathbb{R}^{s+1}\right): f=0 \quad \text { on } \quad F^{c}\right\},
$$

where $F^{c}$ is the complement of $F$. The examples in quantum field theory suggest that $f \in \mathfrak{Q}(F)$ should mean that the Cauchy data of $f$ have support in $F$. Hence we define a net $\Gamma_{c}(\mathscr{A})$ over $\mathscr{C}$ by setting

$$
\Gamma_{c}(\mathscr{A})(F)=\left\{f \in \mathscr{A}\left(\mathbb{R}^{s+1}\right): f=0 \quad \text { on } \quad F^{\prime}\right\} .
$$

Here $F^{\prime}$ is the spacelike complement of $F$

$$
F^{\prime}=\left\{x^{\prime} \in \mathbb{R}^{s+1}:\left(x-x^{\prime}\right)^{2}<0, x \in F\right\} .
$$

Clearly $\Gamma_{b}$ and $\Gamma_{c}$ are functors from sheaves to nets.

In this section we develop methods for computing the low dimensional cohomology groups for nets of the form $\Gamma_{b}(\mathscr{A})$ and $\Gamma_{c}(\mathscr{A})$. We shall concentrate on the more complicated case $\Gamma_{c}(\mathscr{A})$, the statements and proofs for $\Gamma_{b}(\mathscr{A})$ can be obtained by replacing the spacelike complement by the complement, the number of space dimensions $s$ by $d=s+1$ and causally soft by soft. In the case of Theorem 2.10 , the proof could also be simplified.

If $U$ is a bounded open set and $\mathscr{O} \in \mathscr{K}_{0}$ we may choose $a \in \Sigma_{0}$ with $U \subset(\mathcal{O}+a)^{\prime}$ so it follows from (1.7) that

$$
H^{0}\left(\Gamma_{c}(\mathscr{A})\right)=0 .
$$

Actually, it is not difficult to show that if $s>1$, then $H^{1}\left(\Gamma_{c}(\mathscr{A})\right)=0$. We give a deliberately pedestrian proof because it helps to illuminate the necessarily more technical discussion of $H^{2}\left(\Gamma_{c}(\mathscr{A})\right)$.

If $\mathscr{O} \in \mathscr{K}_{0}, c \in \Sigma_{n}$, we denote $(|c|+\mathcal{O})^{\prime}$ by $\Omega_{c}(\mathcal{O})$ or even $\Omega_{c}$ if $\mathscr{O}$ can be understood from the context.

2.1. Lemma. If $z \in Z^{1}\left(\mathcal{C} ; \Gamma_{c}(\mathscr{A})\right)$ and $s \neq 1$ then $z(b)=0$ on $\Omega_{\widehat{c}_{0} b}(\mathcal{O}) \cap \Omega_{\hat{\tau}_{1} b}(\mathcal{O}), b \in \Sigma_{1}$.

Proof. The cocycle identity implies that

$$
z\left(\partial_{0} c\right)=0 \quad \text { on } \quad \Omega_{\hat{\sigma}_{1} c} \cap \Omega_{\hat{\sigma}_{2} c}, c \in \Sigma_{2} .
$$


Now $x \in \Omega_{\hat{\sigma}_{0} b} \cap \Omega_{\hat{c}_{1} b}$ is equivalent to $\partial_{0} b, \partial_{1} b \in \Omega_{x}$. If $s>1, \Omega_{x}$ is path-connected. Hence there is a $c \in \Sigma_{2}$ with $\partial_{0} c=b$ and $x \in \Omega_{\partial_{1} c} \cap \Omega_{\partial_{2} c}$. Thus $\Omega_{\hat{\sigma}_{0} b} \cap \Omega_{\hat{\sigma}_{1} b} \subset \bigcup_{\hat{c}_{0} c=b} \Omega_{\hat{\sigma}_{1} c} \cap \Omega_{\hat{\partial}_{2} \mathrm{c}}$ and this proves the lemma.

2.2. Theorem. If $s>1$, then $H^{1}\left(\Gamma_{c}(\mathscr{A})\right)=0$.

Proof. Let $z \in Z^{1}\left(\mathcal{O} ; \Gamma_{c}(\mathscr{A})\right)$; if $z=d y$ with $y \in C^{0}\left(\mathscr{C} ; \Gamma_{c}(\mathscr{A})\right)$

$$
z(b)-y\left(\partial_{0} b\right)=0 \quad \text { on } \Omega_{\widehat{c}_{1} b}, b \in \Sigma_{1} .
$$

We first show that there is a unique $a \rightarrow y(a) \in \mathscr{A}\left(\mathbb{R}^{s+1}\right)$ satisfying (2.4). Since $\left\{\Omega_{\partial_{1} b}: b \in \Sigma_{1}, \partial_{0} b=a\right\}$ is an open covering of $\mathbb{R}^{s+1}$ and $\mathscr{A}$ is a sheaf, it suffices to show that if $\partial_{0} b=\partial_{0} b^{\prime}$

$$
z(b)=z\left(b^{\prime}\right) \text { on } \quad \Omega_{\hat{o}_{1} b} \cap \Omega_{\hat{\partial}_{1} b^{\prime}} .
$$

However, given $b, b^{\prime} \in \Sigma_{1}$ with $\partial_{0} b=\partial_{0} b^{\prime}$, there exists a $c \in \Sigma_{2}$ with $\partial_{0} c=b$ and $\partial_{1} c=b^{\prime}$. Applying the cocycle identity and Lemma 2.1, we deduce (2.5) as required. Taking $b=\sigma_{0} a$ in $(2.4)$, we see that $y \in C^{0}\left(\mathcal{O} ; \Gamma_{c}(\mathscr{A})\right)$. It remains to show that $z=d y$. Given $b \in \Sigma_{1}$, pick $c \in \Sigma_{2}$ with $\partial_{0} c=b$ and $\partial_{0} b, \partial_{1} b \in \Omega_{\partial_{1} \partial_{2} c}$. Applying the cocycle identity and (2.4), we deduce

$$
z(b)-y\left(\partial_{0} b\right)+y\left(\partial_{1} b\right)=0 \text { on } \Omega_{\hat{\partial}_{1} \hat{o}_{2} c} .
$$

Since $\left\{\Omega_{\hat{\partial}_{1} \hat{c}_{2} c}: \partial_{0} c=b, \partial_{0} b, \partial_{1} b \in \Omega_{\hat{\partial}_{1} \partial_{2} c}\right\}$ covers $\mathbb{R}^{s+1}$ this implies that $z=d y$ completing the proof.

The restriction $s>1$ is essential here, see Theorem 2.7. The above proof is based on [4, Theorem 2.2], mutatis mutandis. Closer inspection shows that singular homology theory is one of the ingredients. For this reason, we shall consider an $n$ cochain not just as a map from $\Sigma_{n}$ but as a homomorphism from $C_{n} \equiv C_{n}\left(\mathbb{R}^{s+1}\right)$, the group of $n$-chains on Minkowski space. If $b=\sum_{i=1}^{m} \mu_{i} b_{i}$ with $b_{i} \in \Sigma_{n}$ and $\mu_{i} \in \mathbb{Z}$, $\mu_{i} \neq 0$, we set

$$
\Omega_{b}(\mathcal{O})=\bigcap_{i=1}^{m} \Omega_{b_{i}}(\mathcal{O}) .
$$

In other words $\Omega_{b}(\mathcal{O})=(\mathcal{O}+|b|)^{\prime}$ where $|b|=\bigcup_{i=1}^{m} b_{i}\left(\Delta^{n}\right)$ is the support of the chain $b$. Note that $x \in \Omega_{b}(\mathcal{O})$ is equivalent to $b \in C_{n}\left(\Omega_{x}(\mathcal{O})\right)$. Clearly if $y \in C^{n}\left(\mathcal{O} ; \Gamma_{c}(\mathscr{A})\right)$ then

$$
y(b)=0 \quad \text { on } \quad \Omega_{b}(\mathcal{C}), b \in C_{n} .
$$

We adopt the standard notation $B_{n}, Z_{n}$, and $H_{n}$ to denote boundaries, cycles and homology classes. The homology class of $c \in Z_{n}$ is denoted by $[c]$ and the boundary operation is denoted by $\partial$

$$
\partial c=\sum_{i=0}^{n}(-1)^{i} \partial_{i} c, \quad c \in \Sigma_{n}
$$

A contracting homotopy for the chain complex $C_{*}$ is a homomorphism $h$ of the $C_{*}$ such that $1=h \partial+\partial h$. 
The following simple Lemma is used repeatedly.

2.3. Lemma. Let $b \in B_{n}$ then if $n \neq s-1$

$$
\bigcup_{\partial c=b} \Omega_{c}(\mathcal{O})=\Omega_{b}(\mathcal{O})
$$

Proof. $x \in \Omega_{b}$ implies $b \in C_{n}\left(\Omega_{x}\right)$ and thus $b \in Z_{n}\left(\Omega_{x}\right)$. Now $\Omega_{x}$ is homeomorphic to the $(s-1)$-sphere, thus $H_{n}\left(\Omega_{x}\right)=0$ if $n \neq 0, s-1$. We deduce that, if $n \neq 0$, there exists $c^{\prime} \in C_{n+1}\left(\Omega_{x}\right)$ with $\partial c^{\prime}=b$. This is even true if $n=0$ because if $\varepsilon: C_{0}\left(\Omega_{x}\right) \rightarrow \mathbb{Z}$ denotes the canonical augmentation, $\varepsilon(b)=0$ since $b \in B_{0}$. However $x \in \Omega_{c^{\prime}}$ so $\Omega_{b}=\bigcup_{\partial c=b} \Omega_{c}$ as required.

We now generalize Lemma 2.1

2.4. Corollary. If $z \in Z^{n}\left(\mathcal{O} ; \Gamma_{c}(\mathscr{A})\right)$ and $s \neq n$ then

$$
z(c)=0 \quad \text { on } \Omega_{\partial c}(\mathcal{O}), c \in C_{n} .
$$

Proof. If $n=0$ the result follows from (2.3). For $n>0$, let $b=\partial c$ then if $\partial c^{\prime}=b$, $\partial\left(c-c^{\prime}\right)=0$ and since $H_{n}\left(\mathbb{R}^{s+1}\right)=0$ and $z$ is an $n$-cocycle $z(c)=z\left(c^{\prime}\right)$. Thus by (2.7), $z(c)=0$ on $\bigcup_{\partial c^{\prime}=b} \Omega_{c^{\prime}}=\Omega_{b}$.

If $s=n$, what we get instead is

$$
z(c)=0 \text { on }\left\{x \in \Omega_{\partial c}(\mathcal{O}):[\partial c]=0 \text { in } H_{s-1}\left(\Omega_{\lambda}(\mathcal{O})\right\} .\right.
$$

However since (2.8) is satisfied if $z \in B^{s}\left(\mathcal{O} ; \Gamma_{c}(\mathscr{A})\right)$, we can begin to analyse $H^{s}\left(\Gamma_{c}(\mathscr{A})\right)$. To this end, we pick a coherent set of generators for the $H_{s-1}\left(\Omega_{x}\right)$. Let $c_{1}, c_{2}, \ldots, c_{n}, \ldots$ be a sequence of concentric $s$-balls in, say, the hyperplane $x^{0}=0$, whose radii increase monotonically to infinity. We may consider the $c_{i}$ as $s$-chains after orienting them so that their normal is in the direction of the positive $x^{0}$-axis. Given $x \in \mathbb{R}^{s+1}$, there exists an $i_{\lambda}$ such that $\left[\partial c_{1}\right]$ is a generator for $H_{s-1}\left(\Omega_{\lambda}\right)$, if and only if $i \geqq i_{x}$ and $\left[\partial c_{t}-\partial c_{j}\right]=0$ for $i, j \geqq i_{x}$. Given $z \in Z^{s}\left(\mathcal{O} ; \Gamma_{c}(\mathscr{A})\right)$, we define $\gamma(z) \in \mathscr{A}\left(\mathbb{R}^{s+1}\right)$ by

$$
\gamma(z)=z\left(c_{i}\right) \text { on } \quad\left\{x \in \Omega_{\hat{c}_{c_{1}}}: i \geqq i_{x}\right\} .
$$

(2.9) shows that this definition is consistent.

2.5. Proposition. $\gamma: Z^{s}\left(\mathcal{O} ; \Gamma_{c}(\mathscr{A})\right) \rightarrow \mathscr{A}\left(\mathbb{R}^{s+1}\right)$ is a homomorphism and $\gamma(z)=0$ if and only if (2.8) holds (with $s=n) . \gamma$ extends to homomorphism of $Z^{s}\left(\Gamma_{c}(\mathscr{A})\right.$ ) into $\mathscr{A}\left(\mathbb{R}^{s+1}\right)$ and factors to give a homomorphism $\hat{\gamma}: H^{s}\left(\Gamma_{c}(\mathscr{A})\right) \rightarrow \mathscr{A}\left(\mathbb{R}^{s+1}\right)$. If $s=1, \hat{\gamma}$ is a monomorphism.

Proof. Clearly $\gamma$ is a homomorphism and (2.8) implies $\gamma(z)=0$. Conversely if $\gamma(z)=0$ and $x \in \Omega_{\hat{\sigma} c}$ then for $i \geqq i_{x},\left[\partial c-n \partial c_{i}\right]=0$ in $H_{s-1}\left(\Omega_{x}\right)$ for some $n \in \mathbb{Z}$. Hence $z(c)=n z\left(c_{l}\right)=n \gamma(z)=0$ on some neighbourhood of $x . \gamma(z)$ is unchanged if $z$ is considered as an element of $Z^{s}\left(\mathcal{O}_{1} ; \Gamma_{c}(\mathscr{A})\right)$ for $\mathcal{O}_{1} \supset \mathcal{O}$ so $\gamma$ extends to a homomorphism of $Z^{s}\left(\Gamma_{c}(\mathscr{A})\right)$ which factors to a homomorphism $\hat{\gamma}$ of $H^{s}\left(\Gamma_{c}(\mathscr{A})\right)$ into $\mathscr{A}\left(\mathbb{R}^{s+1}\right)$ since coboundaries satisfy (2.8). If $s=1$ and $\gamma(z)=0$ we may apply the argument of Theorem 2.2 to show that $z \in B^{1}\left(\mathcal{O} ; \Gamma_{c}(\mathscr{A})\right)$. Hence $\hat{\gamma}$ is a monomorphism if $s=1$. 
2.6. Definition. A sheaf $\mathscr{A}$ is causally soft if given any spacelike hyperplane $\mathrm{H}$ and any closed set $\mathrm{F}$ which is the union of double cones based on $\mathrm{H}$, every section of $\mathscr{A}$ over $F$ can be extended to a global section.

Of course every soft sheaf is causally soft but it is sufficient if the restriction of $\mathscr{A}$ to any spacelike hyperplane $\mathrm{H}$ is soft and if, for $\mathrm{F}$ as above, the restriction mapping from $\mathscr{A}(\mathrm{F})$ to $\mathscr{A}(\mathrm{F} \cap \mathrm{H})$ is an isomorphism. This is the case if one considers the sheaf of smooth solutions of hyperbolic differential equations with constant coefficients, such as the wave equation or the Klein-Gordon equation, whose characteristic planes are null planes. This can be deduced, for example, from the discussion of the Cauchy problem in [7, Chapter 5].

2.7. Theorem. If $s=1$, and $\mathscr{A}$ is a causally soft sheaf then $\hat{\gamma}: H^{1}\left(\Gamma_{c}(\mathscr{A})\right) \rightarrow \mathscr{A}\left(\mathbb{R}^{2}\right)$ is an isomorphism.

Proof. In view of Proposition 2.5, it sufficies to show that $\gamma$ is surjective. Since $\mathscr{A}$ is causally soft, given $f \in \mathscr{A}\left(\mathbb{R}^{2}\right), \mathscr{O} \in \mathscr{K}_{0}$ and $a \in \Sigma_{0}$, there exists a $y(a) \in \mathscr{A}$ with $y(a)$ $=f$ on $(\mathcal{O}+a)^{l}$ and $y(a)=0$ on $(\mathcal{O}+a)^{r}$. Here $(\mathcal{O}+a)^{l}$ and $(\mathcal{O}+a)^{r}$ are the connected components of $(0+a)^{\prime},(0+a)^{l}$ being defined by the convention that $\left(0, x^{1}\right) \in(0+a)^{l}$ if $x^{1}$ is sufficiently negative. Define $z(b)=y\left(\partial_{0} b\right)-y\left(\partial_{1} b\right)$ for $b \in \Sigma_{1}$, then $z(b)=0$ on $(\mathcal{O}+b)^{\prime}$ so $z \in Z^{1}\left(\mathcal{O} ; \Gamma_{c}(\mathscr{A})\right)$ and we see that $\gamma(z)=f$.

For $H^{2}\left(\Gamma_{c}(\mathscr{A})\right)$, the method of Theorem 2.2 fails because if $b \in \Sigma_{1}, y(b)$ is not uniquely determined by requiring that $z=d y$. However the restriction of $y(b)$ to $\Omega_{\partial b}(\mathcal{C})$ is uniquely determined.

2.8. Lemma. Let $z \in Z^{n}\left(\mathcal{O} ; \Gamma_{c}(\mathscr{A})\right)$, with $\gamma(z)=0$ if $s=n$, then if $s \neq n-1$, there is a unique map $C_{n-1} \ni b \rightarrow y(b) \in \mathscr{A}\left(\Omega_{\partial b}(\mathcal{O})\right)$ with

$$
\begin{aligned}
& y(\hat{o c})=z(c), \\
& y(b)=0 \quad \text { on } \Omega_{b}(\mathcal{O}), \\
& y(b)+y\left(b^{\prime}\right)=y\left(b+b^{\prime}\right) \text { on } \Omega_{\hat{o} b}(\mathcal{O}) \cap \Omega_{\hat{o} b^{\prime}}(\mathcal{O}) .
\end{aligned}
$$

Proof. Suppose $y$ is given, $b \in C_{n-1}$ and $c \in C_{n}$ then

$$
y(b)+y(\partial c-b)=y(\partial c)=z(c) \text { on } \Omega_{\hat{c} b}
$$

by $(2.11)$ and (2.13). Hence by $(2.12)$

$$
y(b)=z(c) \text { on } \Omega_{b-\partial c} .
$$

Since by Lemma 2.3, $\bigcup_{c} \Omega_{b-\hat{c} c}=\Omega_{\partial b}, y$ is unique. Conversely we use (2.14) to define $y$.

$$
\begin{aligned}
& \operatorname{By}(2.8) \\
& z(c)-z\left(c^{\prime}\right)=z\left(c-c^{\prime}\right) \text { on } \Omega_{r\left(c-c^{\prime}\right)}
\end{aligned}
$$

and $\Omega_{\partial\left(c-c^{\prime}\right)} \supset \Omega_{b-\partial c} \cap \Omega_{b-\hat{o} c^{\prime}}$ so (2.14) defines $y(b) \in \mathscr{A}\left(\Omega_{\partial b}\right)$. If $b=\partial c,(2.14)$ reduces to (2.11). Since $z$ is a homomorphism, we have

$$
y(b)+y\left(b^{\prime}\right)=y\left(b+b^{\prime}\right) \text { on } \Omega_{b-\hat{o} c} \cap \Omega_{b^{\prime}-\partial c^{\prime}}
$$

and again Lemma 2.3 gives (2.13). Taking $c=0$ in (2.14) we get (2.12). 
From this Lemma, it is clear that the problem of deciding whether an $n$-cocycle is an $n$-coboundary for $n<s$ is one of extending $y(b)$ to a global section of $\mathscr{A}$ so that $y \in C^{n-1}\left(\Gamma_{c}(\mathscr{A})\right)$. We can again hope that the causal softness of $\mathscr{A}$ might be sufficient to guarantee the triviality of the cohomology.

We first take advantage of the contractibility of Minkowski space to simplify the geometric considerations.

2.9. Lemma. Let $h$ be a contracting homotopy for $C_{*}$, then under the hypotheses of Lemma 2.8, given $a \in B_{n-2}$ pick $b \in C_{n-1}$ with $\partial b=a$ and set

$$
x(a)=y(b)-z(h b) \quad \text { on } \quad \Omega_{a}(\mathcal{C})
$$

then $x(a)$ is independent of the choice of $b$ and

$$
x(a)+x\left(a^{\prime}\right)=x\left(a+a^{\prime}\right) \quad \text { on } \quad \Omega_{a}(\mathcal{O}) \cap \Omega_{a^{\prime}}(\mathcal{O})
$$

and $z \in B^{n}\left(\mathcal{O} ; \Gamma_{c}(\mathscr{A})\right)$ if and only if there exists a $w \in C^{n-2}\left(\mathscr{A}\left(\mathbb{R}^{s+1}\right)\right)$ with

$$
w(a)=x(a) \quad \text { on } \quad \Omega_{a}(\mathcal{O}), a \in B_{n-2} .
$$

Proof. Let $b, b^{\prime} \in C_{n-1}$ with $\partial\left(b-b^{\prime}\right)=0$ then $b-b^{\prime}=\partial h\left(b-b^{\prime}\right)$ and (2.11) and (2.13) show that

$$
y(b)-y\left(b^{\prime}\right)=z\left(h\left(b-b^{\prime}\right)\right) \text { on } \Omega_{\hat{o} b}(\mathcal{O}) .
$$

Hence the right hand side of (2.15) is independent of $b$. (2.15) and (2.13) imply (2.16). If $z \in B^{n}\left(\mathcal{U} ; \Gamma_{c}(\mathscr{A})\right)$ then

$$
z=d y^{\prime} \quad \text { with } \quad y^{\prime} \in C^{n-1}\left(\mathcal{O} ; \Gamma_{c}(\mathscr{A})\right)
$$

so $y^{\prime}(b)-z(h(b)) \in Z^{n-1}\left(\mathscr{A}\left(\mathbb{R}^{s+1}\right)\right)$ and there is a $w \in C^{n-2}\left(\mathscr{A}\left(\mathbb{R}^{s+1}\right)\right)$ with

$$
w(\partial b)=y^{\prime}(b)-z(h b), b \in C_{n-1} .
$$

Since $y^{\prime}(b)$ extends $y(b)$ by Lemma 2.8, (2.17) follows. Conversely given $w \in C^{n-2}\left(\mathscr{A}\left(\mathbb{R}^{s+1}\right)\right)$ satisfying $(2.17)$ define $y^{\prime}(b)$ by (2.18) then $y^{\prime}(b)$ extends $y(b)$ so $y^{\prime} \in C^{n-1}\left(\mathcal{O} ; \Gamma_{c}(\mathscr{A})\right)$ and $z=d y^{\prime}$.

We come now to the main Theorem of this section.

2.10. Theorem. If $s>2$ and $\mathscr{A}$ is a causally soft sheaf then $H^{2}\left(\Gamma_{c}(\mathscr{A})\right)=0$.

Proof. We show that if $z \in Z^{2}\left(\mathcal{O}_{1} ; \Gamma_{c}(\mathscr{A})\right)$ and if $\mathcal{O} \in \mathscr{K}_{0}, \mathcal{O}_{1} \operatorname{Cint} \mathcal{O}$ then there is a $y \in C^{1}\left(\mathcal{O} ; \Gamma_{c}(\mathscr{A})\right)$ with $d y=z$. We may define $x$ as in Lemma 2.9 with $\mathcal{O}=\mathcal{O}_{1}$ and it suffices to find $w \in C^{0}\left(\mathscr{A}\left(\mathbb{R}^{s+1}\right)\right)$ with

$$
x(\partial b)=w(\partial b) \quad \text { on } \quad \Omega_{\partial b}(\mathcal{O}), b \in \Sigma_{1},
$$

since every $a \in B_{0}$ can be written in the form $a=\sum_{i=1}^{k} \partial b_{i}$ with $b_{i} \in \Sigma_{1}$ and $|a|$ $=\bigcup_{i=1}^{k}\left|\partial b_{i}\right|$. Furthermore, since $\mathscr{A}$ is a causally soft, it is sufficient to show that there is a function $\Sigma_{0} \ni a \rightarrow w(a) \in \mathscr{A}\left(\Omega_{a}(\mathcal{O})\right)$ with

$$
w\left(\partial_{0} b\right)-w\left(\partial_{1} b\right)=x(\partial b) \quad \text { on } \quad \Omega_{\hat{o}_{0} b}(\mathcal{O}) \cap \Omega_{\hat{o}_{1} b}(\mathcal{O}), b \in \Sigma_{1} .
$$


Suppose we have defined $w(a)$ for $a \in X \subset \Sigma_{0}$ such that (2.19) holds whenever $\partial_{0} b, \partial_{1} b \in X$. Given $a \in \Sigma_{0}, a \notin X,(2.16)$ implies that for $a^{\prime}, a^{\prime \prime} \in X$

$$
w\left(a^{\prime}\right)+x\left(a-a^{\prime}\right)=w\left(a^{\prime \prime}\right)+x\left(a-a^{\prime \prime}\right) \quad \text { on } \quad \Omega_{a}(\mathcal{O}) \cap \Omega_{a^{\prime}}(\mathcal{O}) \cap \Omega_{a^{\prime \prime}}(\mathcal{O}) .
$$

Thus there exists a $v \in \mathscr{A}(U(X, a, \mathcal{O}))$ where

$$
\begin{aligned}
& U(X, a, \mathcal{O})=\bigcup_{a^{\prime} \in X} \Omega_{a}(\mathcal{O}) \cap \Omega_{a^{\prime}}(\mathcal{O}) \\
& w\left(\partial_{0} b\right)-w\left(\partial_{1} b\right)=x(\partial b) \quad \text { on } \quad \Omega_{\hat{\partial}_{0} b}(\mathcal{O}) \cap \Omega_{\hat{c}_{1} b}(\mathcal{O}), b \in \Sigma_{1} .
\end{aligned}
$$

Suppose we have defined $w(a)$ for $a \in X \subset \Sigma_{0}$ such that (2.19) holds whenever $\partial_{0} b, \partial_{1} b \in X$. Given $a \in \Sigma_{0}, a \notin X,(2.16)$ implies that for $a^{\prime}, a^{\prime \prime} \in X$

$$
w\left(a^{\prime}\right)+x\left(a-a^{\prime}\right)=w\left(a^{\prime \prime}\right)+x\left(a-a^{\prime \prime}\right) \quad \text { on } \quad \Omega_{a}(\mathcal{O}) \cap \Omega_{a^{\prime}}(\mathcal{O}) \cap \Omega_{a^{\prime \prime}}(\mathcal{O}) .
$$

Thus there exists a $v \in \mathscr{A}(U(X, a, \mathcal{O}))$ where

$$
U(X, a, \mathcal{O})=\bigcup_{a^{\prime} \in X} \Omega_{a}(\mathcal{O}) \cap \Omega_{a^{\prime}}(\mathcal{O})
$$

with $v=w\left(a^{\prime}\right)+x\left(a-a^{\prime}\right)$ on $\Omega_{a}(\mathcal{O}) \cap \Omega_{a^{\prime}}(\mathcal{O})$. To define $w(a)$ so that (2.19) holds for $\partial_{0} b, \partial_{1} b \in X \cup\{a\}$ we have only to extend $v$ to $\Omega_{a}(\mathcal{O})$. If $U(X, a, \mathcal{O})=\Omega_{a}(\mathcal{O})$, there is nothing to be done and if $U$ is the union of double cones based on some spacelike hyperplane $H$, we may appeal to the causal softness of $\mathscr{A}$ to extend $v$ at the cost of redefining $\mathcal{O}$. We therefore proceed as follows. Let $\mathcal{O}_{1}$ be based on the spacelike hyperplane $H$. Pick $a_{1}, a_{2}, a_{3}, \ldots$ on $H$ tending spacelike to infinity and $\mathcal{O}_{i} \in \mathscr{K}_{0}$ based on $H$ with $\mathcal{O}_{i} \subset \operatorname{int} \mathcal{O}_{i+1} \subset \mathcal{O}$. Set $X_{1}=\left\{a_{1}\right\}$ and $X_{i+1}=X_{i} \cup\left\{a_{i+1}\right\}$. Then $U\left(X_{i}, a_{i+1}, \mathcal{O}_{i}\right)$ is the union of double cones based on $H$ and $U(X, a, \mathcal{O})=\Omega_{a}(\mathcal{O})$ provided $X \supset \bigcup_{n} X_{n}$. This completes the proof.

The proof above also shows that when $\mathscr{A}$ is causally soft the map $\hat{\gamma}$ of Proposition 2.5 is a monomorphism for $s=2$. Presumably $\hat{\gamma}$ is even an isomorphism in this case but before tackling either this problem or the problem of higher cohomologies, it would seem advisable to develop more powerful methods.

In this section the sheaf $\mathscr{A}$ has been treated as the primary object. In the longer term it would be desirable to have results expressed directly in terms of properties of the nets involved.

\section{Nets over $\mathscr{K}$}

We now consider the cohomology of nets which are defined just on double cones and not on arbitrary compact sets. If $\mathscr{U}$ is a net over $\mathscr{K}$ we cannot define cochains directly using (1.1). Instead we first extend $\mathfrak{U}$ to a net over $\mathscr{C}$. This may, in general, be done in many different ways; in particular we can take the minimal extension defined by

$$
\tilde{\mathfrak{U}}(F)=\vee\{\mathfrak{U}(\mathcal{O}): \mathcal{O} \subset F\},
$$

where $V$ denotes the least upper bound in the lattice of subgroups of the group $\cup\{\mathfrak{U}(\mathcal{O}): \mathcal{O} \in \mathscr{K}\}$. Our aim is to show that the cohomology is independent of the extension and is thus an intrinsic property of the net $\mathfrak{A}$. This is easily proved using barycentric decompositions, a standard technique of algebraic topology. 
A mapping $\phi: C_{*} \rightarrow C_{*}$ is said to be local if $|\phi(c)| C|c|$, i.e. if $\phi$ reduces supports. The importance of such mappings is that if $\mathfrak{A}$ is a net over $\mathscr{C}$, there is an induced mapping $\phi^{*}: C^{*}(\mathfrak{U}) \rightarrow C^{*}(\mathfrak{U})$ defined by

$$
\phi^{*}(f)(c)=f(\phi(c)), c \in C_{*} .
$$

Indeed net cohomology owes its existence to the fact that $\partial$ is local.

If $c \in \Sigma_{n}$, we let $k(c) \in \Sigma_{n+1}$ be the cone on the barycentre of $c$

$$
k(c)\left(t^{0}, t^{1}, \ldots, t^{n+1}\right)=c\left(\frac{1}{n+1} t^{0}+t^{1}, \frac{1}{n+1} t^{0}+t^{2}, \ldots, \frac{1}{n+1} t^{0}+t^{n+1}\right) .
$$

Since $k(c)\left(\Delta^{n+1}\right)=c\left(\Delta^{n}\right), k$ extends to give a local homomorphism of degree 1 of $C_{*}$ and thus $u: C_{*} \rightarrow C_{*}$ defined by

$$
u=1-k \hat{\partial}-\partial k
$$

is a local chain endomorphism of $\boldsymbol{C}_{*}$ called the barycentric decomposition.

Let $\mathfrak{M}$ be a covering of Minkowski space such that each point is in the interior of some element of $\mathfrak{M}$. A simplex is said to be small of order $\mathfrak{M}$ if its support is contained in some element of $\mathfrak{M}$. Let $C_{*}(\mathfrak{M})$ be the subcomplex of $C_{*}$ generated by these simplexes. If $c \in \Sigma_{*}$ let $n(c)$ denote the least integer such that $u^{n(c)}(c) \in C_{*}(\mathfrak{M})$ and define

$$
h(c)=k\left(1+u+\ldots+u^{n(c)-1}\right)(c)
$$

then $h$ extends to give a local homomorphism of degree 1 of $C_{*}$ and we get a local chain endomorphism $v$ by setting

$$
v=1-h \partial-\partial h .
$$

As is well known $[8, \S 8] v$ is a projection mapping of $\boldsymbol{C}_{*}$ onto $\boldsymbol{C}_{*}(\mathfrak{M})$.

If $\mathfrak{U}$ is a net over $\mathscr{C}$, we can define a cochain complex, $C_{\mathfrak{M}}^{*}(\mathfrak{U})$, in the obvious way: $f \in C_{\mathfrak{M}}^{n}(\mathfrak{U})$ is a homomorphism $f: C_{n}(\mathfrak{M}) \rightarrow \bigcup_{F \in \mathscr{C}} \mathfrak{A}(F)$ such that there exists an $\mathcal{O} \in \mathscr{K}_{0}$ with

$$
f(c) \in \mathfrak{A}(\mathcal{O}+|c|), c \in C_{n}(\mathfrak{M}) .
$$

Since $v$ is local, it induces an injection $v^{*}: C_{\mathfrak{M}}^{*}(\mathfrak{U}) \rightarrow C^{*}(\mathfrak{U})$ which is a right inverse for the restriction map $C^{*}(\mathfrak{U}) \rightarrow C_{\mathfrak{M}}^{*}(\mathfrak{U})$. In virtue of the homotopy formule (3.6), $\boldsymbol{H}_{\mathfrak{M}}^{*}(\mathfrak{Q})$ and $\boldsymbol{H}^{*}(\mathfrak{U})$ are isomorphic.

3.1. Theorem. Let $\mathfrak{A}$ and $\mathfrak{B}$ be nets over $\mathscr{C}$ whose restrictions to $\mathscr{K}$ agree then $\boldsymbol{H} *(\mathfrak{U})$ $=\boldsymbol{H} *(\mathfrak{B})$.

Proof. Without loss of generality we may suppose by (3.1) that $\mathfrak{A}(F) \subset \mathfrak{B}(F), F \in \mathscr{C}$. Then $C_{\mathfrak{M}}^{*}(\mathfrak{U}) \subset C_{\mathfrak{M}}^{*}(\mathfrak{B})$.

The result follows if we show that for a covering of the form $\mathfrak{M}=\{\mathbb{O}$ $\left.+x: x \in \mathbb{R}^{s+1}\right\}$, where $\mathcal{O} \in \mathscr{K}_{0}, C_{\mathfrak{M}}^{*}(\mathfrak{U})=C_{\mathfrak{M}}^{*}(\mathfrak{B})$. If $f \in C_{\mathfrak{M}}^{n}\left(\mathcal{O}_{1} ; \mathfrak{B}\right)$, pick $\mathcal{O}_{2}, \mathcal{O}_{3} \in \mathscr{K}_{0}$ such that $\mathcal{O}_{2} \supset \mathcal{O}+\mathcal{O}_{1}$ and $\mathcal{O}_{3} \supset \mathcal{O}+\mathcal{O}_{2}$. Let $c \in \Sigma_{n}$ be $\mathfrak{M}$-small, then $|c| c \mathcal{O}+x$ for some $x$, so $f(c) \in \mathfrak{B}\left(\mathcal{O}_{1}+|c|\right) \subset \mathfrak{B}\left(\mathcal{O}_{2}+x\right)=\mathfrak{U}\left(\mathcal{O}_{2}+x\right) \subset \mathfrak{U}\left(\mathcal{O}_{3}+|c|\right)$ thus $f \in C_{\mathfrak{M}}^{n}\left(\mathcal{O}_{3} ; \mathfrak{U}\right)$ as required. 
Shed of its technicalities, the proof may be summarized as follows : if $z \in Z^{n}(\mathfrak{B})$ then $z^{\prime}$ defined by

$$
z^{\prime}(c)=z(v(c)), c \in C_{n}
$$

is a cohomologous cocycle in the subgroup $Z^{n}(\mathfrak{U})$.

In view of Theorem 3.1, we may speak of the cohomology of a net over $\mathscr{K}$.

3.2. Theorem. Let $0 \rightarrow \mathfrak{A} \stackrel{\phi}{\longrightarrow} \mathfrak{B} \stackrel{\psi}{\longrightarrow} \mathfrak{C} \rightarrow 0$ be an exact sequence of nets over $\mathscr{K}$, i.e.

$$
0 \rightarrow \mathfrak{U}(\mathcal{O}) \rightarrow \mathfrak{B}(\mathcal{O}) \rightarrow \mathfrak{C}(\mathcal{O}) \rightarrow 0
$$

is exact for each $\mathcal{O} \in \mathscr{K}$. Then we have an induced long exact sequence in cohomology

$$
0 \rightarrow H^{0}(\mathfrak{U}) \rightarrow H^{0}(\mathfrak{B}) \rightarrow H^{0}(\mathfrak{C}) \rightarrow H^{1}(\mathfrak{U}) \rightarrow H^{1}(\mathfrak{B}) \rightarrow \ldots .
$$

Proof. Extend $\mathfrak{B}$ to a net over $\mathscr{C}$ and define

$$
\mathfrak{U}(F)=\phi^{-1}(\mathfrak{B}(F)), \mathfrak{C}(F)=\psi(\mathfrak{B}(F)), F \in \mathscr{C} .
$$

In this way we get an exact sequence of nets over $\mathscr{C}$ so that the result follows from Lemma 1.1.

Theorems 3.1 and 3.2 would still hold if $\mathscr{K}$ is replaced by any subset $\mathscr{L}$ of $\mathscr{C}$ of the form $\mathscr{L}=\left\{L+x: L \in \mathscr{L}_{0}, x \in \mathbb{R}^{s+1}\right\}$ where $\mathscr{L}_{0}$ is a cofinal subset of $\mathscr{C}$. The choice of $\mathscr{K}$ here is dictated by convenience because for many of the nets which arise in practice we get exact sequences over $\mathscr{K}$ but not over $\mathscr{C}$. We can simplify matters further by introducing the concept of almost exactness. We say that $0 \rightarrow \mathfrak{U} \rightarrow \mathfrak{B} \rightarrow \mathfrak{C} \rightarrow 0$ is almost exact if $0 \rightarrow \breve{\mathfrak{U}} \rightarrow \mathfrak{\mathfrak { B }} \rightarrow \check{\mathfrak{C}} \rightarrow 0$ is exact, where $\mathfrak{\mathfrak { U }}$ denotes the inner regularization of $\mathfrak{A}$.

$$
\check{\mathfrak{U}}(\mathcal{O})=\cup\left\{\mathfrak{U}\left(\mathcal{O}_{1}\right): \mathcal{O}_{1} \operatorname{cint} \mathcal{O}, \mathcal{O}_{1} \in \mathscr{K}\right\} .
$$

Any exact sequence of nets is almost exact and an almost exact sequence still gives rise to a long exact sequence in cohomology since $\boldsymbol{H}^{*}(\check{\mathfrak{U}})=\boldsymbol{H}^{*}(\mathfrak{U})$ as we remarked in Section 1. In the next section we shall prove that various sequences are almost exact and evade the more delicate, but irrelevant, issue of exactness.

\section{Computing Net Cohomology}

Net cohomology is computed on the same lines as other cohomology theories. Simple cases, such as the cohomology of trivial nets (Section 1), are computed directly. Then criteria are developed for cohomologies to be trivial; we already have Theorems 2.2 and 2.10 at our disposal. Finally more complicated cohomologies are computed using exact sequences.

Most of our examples of exact sequences rely directly or indirectly on the homology or cohomology of subsets of Minkowski space. Thus the singular chain groups $\mathcal{O} \rightarrow C_{n}(\mathcal{O})$ give rise to a net over $\mathscr{K}$ by identifying $C_{n}(\mathcal{O})$ with $\left\{c \in C_{n}:|c| \subset \mathcal{O}\right\}$. 
Now combining the exact sequences

$$
0 \rightarrow Z_{n}(\mathcal{O}) \rightarrow C_{n}(\mathcal{O}) \stackrel{\partial}{\longrightarrow} B_{n-1}(\mathcal{O}) \rightarrow 0
$$

with $H_{n}(\mathcal{O})=0$ for $n>0$ and $H_{0}(\mathcal{O})=\mathbb{Z}$, we have exact sequences

$$
0 \rightarrow B_{0}(\mathcal{O}) \rightarrow C_{0}(\mathcal{O}) \stackrel{\varepsilon}{\longrightarrow} \mathbb{Z} \rightarrow 0,
$$

where $\varepsilon$ is the augmentation, and

$$
0 \rightarrow B_{n}(\mathcal{O}) \rightarrow C_{n}(\mathcal{O}) \stackrel{\hat{o}}{\longrightarrow} B_{n-1}(\mathcal{O}) \rightarrow 0 \quad n \geqq 1 .
$$

Since $\partial C_{n+1}(\mathcal{O})=B_{n} \cap C_{n}(\mathcal{O}), \mathcal{O} \in \mathscr{K}$, we can regard $\mathcal{O} \rightarrow B_{n}(\mathcal{O})$ as the subnet of $\mathcal{O} \rightarrow C_{n}(\mathcal{O})$ consisting of boundaries and (4.1) and (4.2) allow one to compute the cohomology of the net of boundaries in terms of the cohomology of the net of chains using Theorem 3.2. A direct computation of the low dimensional cohomologies of the net of chains would take us too far afield but the above serves to motivate the next example.

We define a net $\mathscr{E}_{p}^{\prime}$ by letting $\mathscr{E}_{p}^{\prime}(\mathcal{O})$ denote the courants of dimension $p$ with support in $\mathcal{O}$ and let $\mathscr{B}_{p}^{\prime}$ and $\mathscr{Z}_{p}^{\prime}$ denote the subnets of boundaries and cycles, then $\mathscr{Z}_{p}^{\prime}=\mathscr{B}_{p}^{\prime}$ if $p \geqq 1$ and in place of (4.1) and (4.2) we have

$$
\begin{aligned}
& 0 \rightarrow \mathscr{B}_{0}^{\prime} \rightarrow \mathscr{E}_{0}^{\prime} \stackrel{\varepsilon}{\longrightarrow} \mathbb{R} \rightarrow 0 \\
& 0 \rightarrow \mathscr{B}_{p}^{\prime} \rightarrow \mathscr{E}_{p}^{\prime} \stackrel{\hat{0}}{\longrightarrow} \mathscr{B}_{p-1}^{\prime} \rightarrow 0, \quad p \geqq 1 .
\end{aligned}
$$

These sequences are now almost exact over $\mathscr{K}$ as is seen by considering the de Rham homology with compact support of int $\mathcal{O}$. Since $\mathscr{E}_{p}^{\prime}=\Gamma_{b}\left(\mathscr{D}_{p}^{\prime}\right)$, where $\mathscr{D}_{p}^{\prime}$ denotes the sheaf of courants of dimension $p$ and $\mathscr{D}_{p}^{\prime}$ is a soft sheaf, Theorem 3.2 and the results of Section 2 give:

$$
\begin{aligned}
& H^{0}\left(\mathscr{E}_{p}^{\prime}\right)=H^{0}\left(\mathscr{B}_{p}^{\prime}\right)=0 \\
& H^{1}\left(\mathscr{E}_{p}^{\prime}\right)=0, H^{1}\left(\mathscr{B}_{0}^{\prime}\right) \simeq \mathbb{R}, H^{1}\left(\mathscr{B}_{p}^{\prime}\right)=0, p \neq 0 ; d>1 \\
& H^{1}\left(\mathscr{E}_{0}^{\prime}\right) \simeq \mathscr{D}_{0}^{\prime}(\mathbb{R}), H^{1}\left(\mathscr{E}_{1}^{\prime}\right) \simeq \mathscr{D}_{1}^{\prime}(\mathbb{R}), H^{1}\left(\mathscr{B}_{0}^{\prime}\right) \simeq \mathscr{D}_{1}^{\prime}(\mathbb{R}) ; d=1 \\
& H^{2}\left(\mathscr{E}_{p}^{\prime}\right)=0, H^{2}\left(\mathscr{B}_{p}^{\prime}\right)=0 \text { if } \quad p \neq 1, H^{2}\left(\mathscr{B}_{1}^{\prime}\right) \simeq \mathbb{R} ; d>2 .
\end{aligned}
$$

We can also work in terms of differential forms and define a net $\mathscr{D}^{p}$ by letting $\mathscr{D}^{p}(\mathcal{O})$ denote the smooth forms of degree $p$ with support in $\mathcal{O}$. Let $\mathscr{B}^{p}$ and $\mathscr{Z}^{p}$ denote the subnets of exact and closed forms respectively. Then $\mathscr{B}^{p}=\mathscr{Z}^{p}$ if $p \neq d$ and

$$
\begin{aligned}
& 0 \rightarrow \mathscr{B}^{d} \rightarrow \mathscr{D}^{d} \stackrel{\stackrel{\rho}{\longrightarrow}}{\longrightarrow} \mathbb{R} \rightarrow 0 \\
& 0 \rightarrow \mathscr{B}^{p} \rightarrow \mathscr{D}^{p} \stackrel{d}{\longrightarrow} \mathscr{B}^{p+1} \rightarrow 0 \quad p \neq d,
\end{aligned}
$$

where the map $\mathscr{D}^{d} \stackrel{f}{\longrightarrow} \mathbb{R}$ denotes the integration of a $d$-form over $\mathbb{R}^{d}$. These sequences are almost exact as is seen by considering the de Rham cohomology with compact support of int $\mathcal{O}$. Since $\mathscr{D}^{p}=\Gamma_{b}(\mathscr{E} p)$ where $\mathscr{E}^{p}$ denotes the sheaf of 
smooth forms of degree $p$ and $\mathscr{E}^{p}$ is a soft sheaf, we again have:

$$
\begin{aligned}
& H^{0}\left(\mathscr{D}^{p}\right)=H^{0}\left(\mathscr{B}^{p}\right)=0 \\
& H^{1}\left(\mathscr{D}^{p}\right)=0, H^{1}\left(\mathscr{B}^{p}\right)=0 \quad \text { if } \quad p \neq d, H^{1}\left(\mathscr{B}^{d}\right) \simeq \mathbb{R} ; d>1 \\
& H^{1}\left(\mathscr{D}^{p}\right) \simeq \mathscr{E} p(\mathbb{R}), H^{1}\left(\mathscr{B}^{1}\right) \simeq \mathscr{E}^{\mathscr{O}}(\mathbb{R}) ; d=1 \\
& H^{2}\left(\mathscr{D}^{p}\right)=0, H^{2}\left(\mathscr{B}^{p}\right)=0 \quad \text { if } \quad p \neq d-1, H^{2}\left(\mathscr{B}^{d-1}\right) \simeq \mathbb{R} ; d>2 .
\end{aligned}
$$

We next consider the analogue of (4.4) when $\mathscr{D}^{p}=\Gamma_{b}\left(\mathscr{E}^{p}\right)$ is replaced by $\Gamma_{c}\left(\mathscr{E}^{p}\right)$. Let $\mathscr{B}_{c}^{p}$ and $\mathscr{Z}_{c}^{p}$ denote the subnets of exact and closed forms respectively. We still have $\mathscr{B}_{c}^{p}=\mathscr{Z}_{c}^{p}$ for $p \leqq s$ but now $\mathscr{B}_{c}^{s+1}=\mathscr{Z}_{c}^{s+1}=\Gamma_{c}\left(\mathscr{E}^{s+1}\right)$. As compared with (4.4) we have a characteristic shift in dimension: the obstacle is now the integral of a closed $s$-form over a spacelike hyperplane and we let $\dot{\mathscr{B}}_{c}^{s}$ denote the kernel of this homomorphism of $\mathscr{B}_{c}^{s}$ into $\mathbb{R}$. Then

$$
\begin{aligned}
& 0 \rightarrow \dot{\mathscr{B}}_{c}^{s} \rightarrow \mathscr{B}_{c}^{s} \rightarrow \mathbb{R} \rightarrow 0 \\
& 0 \rightarrow \mathscr{B}_{c}^{s-1} \rightarrow \Gamma_{c}\left(\mathscr{E}^{s-1}\right) \stackrel{d}{\longrightarrow} \dot{\mathscr{B}}_{c}^{s} \rightarrow 0 \\
& 0 \rightarrow \mathscr{B}_{c}^{p} \rightarrow \Gamma_{c}\left(\mathscr{E}^{p}\right) \stackrel{d}{\longrightarrow} \mathscr{B}_{c}^{p+1} \rightarrow 0 \quad p \neq s-1
\end{aligned}
$$

is almost exact. Since this is now a problem of "cohomology with causal supports", we give the simple proof explicitly. Here, as in subsequent proofs of almost exactness, the main point needing attention is the surjectivity condition on the right hand map and to establish the notation used in these proofs we give $\mathscr{O} \in \mathscr{K}$ and pick $\mathcal{O}_{i} \in \mathscr{K}$ with $\mathcal{O}=\mathcal{O}_{0}$ and $\mathcal{O}_{i} \operatorname{Cint} \mathcal{O}_{i+1}$. For the proof at hand $\mathcal{O}_{1}$ is enough. Let $f \in \mathscr{B}^{p+1}(\mathcal{O})$, i.e. $f=d g$ and $d g=0$ on $\mathcal{O}^{\prime}$. Hence if $p \neq s-1$, there is a $(p-1)$ form $h$ on $\mathcal{O}^{\prime}$ with $g=d h$ on $\mathcal{O}^{\prime}$. Thus there is a $(p-1)$-form $h^{\prime}$ on $\mathbb{R}^{s+1}$ such that $g^{\prime}=g-d h^{\prime} \in \Gamma_{c}\left(\mathscr{E}^{p}\right)\left(\mathcal{O}_{1}\right)$ and $d g^{\prime}=f$. If $p=s-1$, we need $f \in \dot{\mathscr{B}}_{c}^{s}(\mathcal{O})$ to be able to draw the same conclusions.

Since $\mathscr{E}^{p}$ is a causally soft sheaf, we may use Theorems $2.2,2.7,2.10$, and 3.2 to deduce

$$
\begin{aligned}
& H^{0}\left(\Gamma_{c}(\mathscr{E} p)\right)=H^{0}\left(\mathscr{B}_{c}^{p}\right)=H^{0}\left(\dot{\mathscr{B}}_{c}^{s}\right)=0 \\
& H^{1}\left(\Gamma_{c}\left(\mathscr{E}^{p}\right)\right)=H^{1}\left(\mathscr{B}_{c}^{p}\right)=0, H^{1}\left(\dot{\mathscr{B}}_{c}^{s}\right) \simeq \mathbb{R}, s>1 \\
& H^{1}\left(\Gamma_{c}\left(\mathscr{E}^{p}\right)\right) \simeq \mathscr{E} p\left(\mathbb{R}^{2}\right), H^{1}\left(\dot{\mathscr{B}}_{c}^{1}\right) \simeq \mathscr{E}^{0}\left(\mathbb{R}^{2}\right), H^{1}\left(\mathscr{B}_{c}^{1}\right) \simeq\left\{f \in \mathscr{E}^{1}\left(\mathbb{R}^{2}\right): d f=0\right\} \quad s=1 \\
& H^{2}\left(\Gamma_{c}\left(\mathscr{E}^{p}\right)\right)=0, H^{2}\left(\mathscr{B}_{c}^{p}\right)=0 \text { if } p \neq s-1, H^{2}\left(\mathscr{B}_{c}^{s-1}\right) \simeq \mathbb{R}, H^{2}\left(\dot{\mathscr{B}}_{c}^{s}\right)=0 \quad s>2 .
\end{aligned}
$$

Turning now to the examples of greater relevance to quantum field theory, let $\mathscr{W}$ denote the net derived from the sheaf of smooth solutions of the wave equation by applying $\Gamma_{c}$. Thus

$$
\mathscr{W}(\mathcal{O})=\left\{f \in \mathscr{E}^{0}\left(\mathbb{R}^{s+1}\right): \square f=0, f(x)=0, x \in \mathcal{O}^{\prime}\right\} .
$$

The following sequence of nets over $\mathscr{K}$ is almost exact:

$$
0 \rightarrow \mathscr{W} \rightarrow \Gamma_{c}\left(\mathscr{E}^{0}\right) \stackrel{\square}{\longrightarrow} \Gamma_{c}\left(\mathscr{E}^{0}\right) \rightarrow 0 .
$$


Given $f \in \Gamma_{c}\left(\mathscr{E}^{0}\right)(\mathcal{O})$, we first pick $g \in \mathscr{E}^{0}\left(\mathbb{R}^{s+1}\right)$ with $\square g=f$ (see for example $\left[9\right.$, Theorem 13.2]) and then $g^{\prime} \in \mathscr{E}^{0}\left(\mathbb{R}^{s+1}\right)$ with $\square g^{\prime}=0$ and $\left(g-g^{\prime}\right)=0$ on $\mathcal{O}_{1}^{\prime}$ by looking at Cauchy data on the spacelike hyperplane through the base of $\mathcal{O}$. We can however compute the cohomology of $\mathscr{W}$ without reference to $(4.7) . H^{0}(\mathscr{W})=0$ trivially and $H^{1}(\mathscr{W})=0$ for $s>1$ by Theorem 2.2 since $\mathscr{W}$ is derived from a sheaf. Since the sheaf is causally soft, $H^{1}(\mathscr{W}) \simeq\left\{f \in \mathscr{E}^{0}\left(\mathbb{R}^{2}\right): \square f=0\right\}$ if $s=1$ by Theorem 2.7 and $H^{2}(\mathscr{W})=0$ if $s>2$ by Theorem 2.10 .

Similar remarks apply to the net $\mathscr{G}$ derived from the Klein-Gordon equation.

$$
\mathscr{G}(\mathcal{O})=\left\{f \in \mathscr{E}^{0}\left(\mathbb{R}^{s+1}\right):\left(\square+m^{2}\right) f=0, f(x)=0, x \in \mathcal{O}^{\prime}\right\} .
$$

Now let $\dot{\mathscr{W}}$ denote the subnet of $\mathscr{W}$ got by imposing the subsidiary condition

$$
\int \dot{f}(0, \boldsymbol{x}) d^{s} \boldsymbol{x}=0
$$

on the solutions of the wave equation. (4.9) is a Lorentz invariant condition; it says that the dual tensor of $d f$ is in $\dot{\mathscr{B}}^{\text {s. Clearly }}$

$$
0 \rightarrow \mathscr{\mathscr { W }} \rightarrow \mathscr{W} \rightarrow \mathbb{R} \rightarrow 0
$$

is exact. Thus $H^{0}(\dot{\mathscr{W}})=0, H^{1}(\dot{\mathscr{W}}) \simeq \mathbb{R}$ if $s>1, H^{1}(\dot{\mathscr{W}}) \simeq \mathbb{R} \times H^{1}(\mathscr{W})$ if $s=1$ and $H^{2}(\dot{\mathscr{W}})=0$ if $s>2$.

Let $\mathscr{V}$ denote the net of smooth vector solutions of the wave equation

$$
\mathscr{V}(\mathcal{O})=\left\{f \in \mathscr{E}^{1}\left(\mathbb{R}^{s+1}\right): \square f=0, f(x)=0, x \in \mathcal{O}^{\prime}\right\} .
$$

and $\mathscr{L}$ the subnet of those solutions satisfying the Lorentz condition

$$
\mathscr{L}(\mathcal{O})=\{f \in \mathscr{V}(\mathcal{O}): \delta f=0\} .
$$

Here $\delta$ is the coderivative so that the Lorentz condition in components reads $\partial_{\mu} f^{\mu}=0$ and we have $\square=d \delta+\delta d$. We show that

$$
0 \rightarrow \mathscr{L} \rightarrow \mathscr{V} \stackrel{\delta}{\longrightarrow} \dot{\mathscr{W}} \rightarrow 0
$$

is almost exact. Given $f \in \mathscr{V}(\mathcal{O})$ let

$$
F(x)=f(0, x) \text { and } \dot{F}(x)=\frac{\partial f}{\partial x^{0}}(0, x)
$$

be the Cauchy data on the spacelike hyperplane through the base of $\mathcal{O}$, which we may suppose to be $x^{0}=0$ without loss of generality. Similarly let $G$ and $\dot{G}$ denote the Cauchy data of $g \in \mathscr{W}(\mathcal{O})$. The equation $\delta f=g$ in terms of Cauchy data reads

$$
\begin{aligned}
& \boldsymbol{\nabla} \cdot \boldsymbol{F}-\dot{F}^{0}=G \\
& \boldsymbol{\nabla} \cdot\left(\dot{\boldsymbol{F}}-\boldsymbol{\nabla} F^{0}\right)=\dot{G} .
\end{aligned}
$$

We see from (4.14) and (4.9) that $f \in \mathscr{W}(\mathcal{O})$ implies $\delta f \in \dot{\mathscr{W}}(\mathcal{O})$. Given $g \in \dot{\mathscr{W}}(\mathcal{O})$, define $\dot{F}^{0}=-G, \boldsymbol{F}=0, F^{0}=0$. Since $\int \dot{G}(\boldsymbol{x}) d^{3} \boldsymbol{x}=0$, we can find $\dot{\boldsymbol{F}}$ with $\dot{\boldsymbol{F}}=0$ outside the base of $\mathcal{O}_{1}$ and $\boldsymbol{\nabla} \cdot \dot{\boldsymbol{F}}=\dot{G}$. Using $F$ and $\dot{F}$ to define $f$, we have $f \in \mathscr{V}\left(\mathcal{O}_{1}\right)$ and $\delta f=g$. Thus (4.13) is almost exact.

It should be noted that, after passing to Cauchy data and taking dual tensors, the almost exactness of (4.10) and (4.13) is essentially that of (4.4). For the 
cohomology of $\mathscr{L}$ we get from $(4.13): H^{0}(\mathscr{L})=0 ; H^{1}(\mathscr{L})=0$ if $s>1$; $H^{1}(\mathscr{L}) \simeq\left\{f \in \mathscr{E}^{1}\left(\mathbb{R}^{2}\right): d f=0, \delta f=0\right\}$ if $s=1$ and $H^{2}(\mathscr{L}) \simeq \mathbb{R}$ if $s>2$. The cohomology classes of $H^{2}(\mathscr{L})$ for $s>2$ can be regarded as being parametrized by an electric charge.

Let $\mathscr{M}$ denote the net of smooth solutions of Maxwell's equations

$$
\mathscr{M}(\mathcal{O})=\left\{f \in \mathscr{E}^{2}\left(\mathbb{R}^{s+1}\right): d f=0, \delta f=0, f(x)=0, x \in \mathcal{O}^{\prime}\right\} .
$$

There is an almost exact sequence for $s>2$

$$
0 \rightarrow \mathscr{W} \stackrel{d}{\longrightarrow} \mathscr{L} \stackrel{d}{\longrightarrow} \mathscr{M} \rightarrow 0
$$

which expresses the way Maxwell's equations are solved using a vector potential. To see this, let $f \in \mathscr{M}(\mathcal{O})$ then by (4.5) for $s \neq 2$ there is a $g \in \Gamma_{c}\left(\mathscr{E}^{1}\right)\left(\mathcal{O}_{1}\right)$ with $f=d g$. However by (4.7), we may find $h \in \Gamma_{c}\left(\mathscr{E}^{0}\right)\left(\mathcal{O}_{2}\right)$ with $\square h=\delta g$ and then $g-d h \in \mathscr{L}\left(\mathcal{O}_{2}\right)$ and $d(g-d h)=f$. Here we must also pay attention to the almost exactness at $\mathscr{L}$. If $f \in \mathscr{L}(\mathcal{O})$ and $d f=0$ then by (4.5) if $s \neq 1$, there is $g \in \mathscr{E}^{0}\left(\mathcal{O}_{1}\right)$ with $d g=f$, but $\square g=\delta d g=\delta f=0$ so $g \in \mathscr{W}\left(\mathcal{O}_{1}\right)$.

For $s=2$, the same reasoning shows that

$$
0 \rightarrow \mathscr{W} \stackrel{d}{\longrightarrow} \mathscr{L} \stackrel{d}{\longrightarrow} \dot{\mathscr{M}} \rightarrow 0
$$

is almost exact where $\dot{\mathscr{M}}=\mathscr{M} \cap \dot{\mathscr{B}}_{c}^{2}$. If $s=1, \mathscr{M}=0$ and $0 \rightarrow \mathscr{W} \stackrel{d}{\longrightarrow} \dot{\mathscr{L}} \rightarrow 0 \rightarrow 0$ is almost exact, where $\dot{\mathscr{L}}=\mathscr{L} \cap \dot{\mathscr{B}}_{c}{ }_{c}$.

To compute the cohomology of $\mathscr{M}$, we cannot use (4.16) but instead proceed as follows: let $\mathscr{J}$ be the net of conserved currents

$$
\mathscr{J}(\mathcal{O})=\left\{j \in \Gamma_{c}\left(\mathscr{E}^{1}\right)(\mathcal{O}): \delta j=0\right\}
$$

and $\dot{\mathscr{J}}$ the subnet defined by

$$
\int j^{0}(0, \boldsymbol{x}) d^{s} \boldsymbol{x}=0 .
$$

Of course $\mathscr{J}$ and $\dot{\mathscr{J}}$ are isomorphic to $\mathscr{B}^{s}$ and $\dot{\mathscr{B}}_{c}^{s}$ respectively by passing to dual tensors in Minkowski space. Now

$$
0 \rightarrow \mathscr{M} \rightarrow \Gamma_{c}\left(\mathscr{E}^{2}\right) \stackrel{d \times \delta}{\longrightarrow} \mathscr{B}_{c}^{3} \times \dot{\mathscr{J}} \rightarrow 0
$$

is almost exact if $s \neq 3$. For given $f \in \mathscr{B}^{3}(\mathcal{O})$ and $j \in \dot{\mathscr{J}}(\mathcal{O})$, we may find $g \in \Gamma_{c}\left(\mathscr{E}^{2}\right)\left(\mathcal{O}_{1}\right)$ with $d g=f$ by (4.5) if $s \neq 3$ and $h \in \Gamma_{c}\left(\mathscr{E}^{1}\right)\left(\mathcal{O}_{2}\right)$ with $\square h=j-\delta g$ by (4.7). Since $d \delta h=-\delta d h+j-\delta g \in \dot{\mathscr{J}}, \delta h \in \dot{\mathscr{W}}\left(\mathrm{O}_{2}\right)$ and by (4.13), we may find $h^{\prime} \in \mathscr{V}\left(\mathcal{O}_{3}\right)$ with $\delta h^{\prime}=\delta h$. Thus $g^{\prime}=g+d h-d h^{\prime} \in \Gamma_{c}\left(\mathscr{E}^{2}\right)\left(\mathcal{O}_{3}\right)$ and $d g^{\prime}=d g=f, \delta g^{\prime}=j$. If $s=3$, the same proof shows that

$$
0 \rightarrow \mathscr{M} \rightarrow \Gamma_{c}\left(\mathscr{E}^{2}\right) \stackrel{d \times \delta}{\longrightarrow} \dot{\mathscr{B}}_{c}^{3} \times \dot{J} \rightarrow 0
$$

is almost exact. For the cohomology of the net $\mathscr{M}$, we now get $H^{0}(\mathscr{M})=0$; $H^{1}(\mathscr{M})=0 ; H^{2}(\mathscr{M}) \simeq \mathbb{R} \times \mathbb{R}$ if $s=3$ and $H^{2}(\mathscr{M}) \simeq \mathbb{R}$ if $s>3$. The second cohomology classes of $\mathscr{M}$ are again parametrized by an electric charge and if $s=3$ there is a magnetic charge as an additional parameter. 
Acknowledgements. During the course of this work we have benefitted from the financial support and hospitality of various Institutions. P.L. wishes to thank the C.E.A. for a "bourse Joliot-Curie"; J.E.R. wishes to thank the C.N.R.S. for a research grant and the Universite d'Aix-Marseille II, U.E.R. scientifique de Luminy, for a Visiting Professorship.

\section{References}

1. Wightman, A.S.: Quelques problèmes mathématiques de la théorie quantique relativiste. 75. Colloques Internationaux du CNRS, Lille 1957. Paris: CNRS 1959

2. Wightman, A. S., Gårding, L. : Fields as operator-valued distributions in quantum field theory. Arkiv Fysik 28, 13 (1964)

3. Haag, R. : Discussion des »axiomes « et des propriétés asymptotiques d'une théorie des champs locale avec particules composées. 75. Colloques Internationaux du CNRS, Lille 1957. Paris: CNRS 1959

4. Roberts, J.E. : Local cohomology and superselection structure. Commun. math. Phys. 51, 107--119 (1976)

5. Roberts, J.E.: A survey of local cohomology. Proceedings of the Conference on Mathematical Problems in Theoretical Physics. Rome 1977. Lecture notes in physics 80. Berlin. Heidelberg, New York: Springer 1978

6. Godement, R.: Topologie algébrique et théorie des faisceaux. Paris: Hermann 1958

7. Hörmander,L. : Linear partial differential operators. Berlin, Gottingen, Heidelberg: Springer 1963

8. Cartan,H.: Séminaire Henri Cartan 1948/1949. Topologie Algébrique. Ecole Normale Supérieure

9. Trèves, F.: Basic linear partial differential equations. New York, London: Academic Press 1975

Communicated by R. Haag

Received May 3, 1978 
